\title{
191. Boltzmann Equation on Some Algebraic Structure Concerning Struggle for Existence
}

\author{
By Yoshiaki IтоH \\ Department of Mathematics, Tokyo Institute of Technology \\ (Comm. by Kinjirô Kunugr, M. J. A., May 12, 1971)
}

V. Volterra had treated the problem of struggle for existence in his book based on the biological interest.

Here we will consider some models of struggle for existence. Although the equations are essentially the same to the Volterra's case, the formulations is different from it. The mathematical formulation is done analogously to the case of Kac's caricature of a Maxwellian gas. The Boltzmann equation on some finite algebraic structure will be mentioned.

Model I.

1) There are three types of particles, $A, B$ and $C$, whose numbers are respectively $n_{A}, n_{B}$ and $n_{C} . \quad n_{A}+n_{B}+n_{C}=n$ is a constant integer.

2) Each particle is in a chaotic bath of like particles. Uniform distribution of colliding pairs is assumed.

3) Particles vary by the following collision rule:<smiles>[CH]C([Hg])([Hg])[Hg]</smiles><smiles>[Hg]C([Hg])([Hg])[Hg]</smiles><smiles></smiles><smiles>[10B-]C([10BH2])([10BH2])[175I]</smiles><smiles>CC(C)(Cl)Cl</smiles><smiles>[CH]C(C)([O-])[Hg]</smiles>

that is to say, $A$ is stronger than $B, B$ is stronger than $C$ and $C$ is stronger than $A$.

Mathematical formulation. On the analogy of Boltzmann's problem, we may have

$$
\begin{aligned}
\frac{\partial}{\partial t} n_{A} & =\frac{n_{A}}{n} n_{A}+\frac{n_{B}}{n} n_{A}+\frac{n_{A}}{n} n_{B}-n_{A} \\
\frac{\partial}{\partial t} n_{B} & =\frac{n_{B}}{n} n_{B}+\frac{n_{C}}{n} n_{B}+\frac{n_{B}}{n} n_{C}-n_{B} \\
\frac{\partial}{\partial t} n_{C} & =\frac{n_{C}}{n} n_{C}+\frac{n_{A}}{n} n_{C}+\frac{n_{C}}{n} n_{A}-n_{C} .
\end{aligned}
$$

(2) is rewritten in the following way for sufficiently large $n$. 


$$
\begin{aligned}
& \left(\frac{n_{A}}{n}, \frac{n_{B}}{n}, \frac{n_{C}}{n}\right) \equiv\left(P_{A}, P_{B}, P_{C}\right) \\
& \frac{\partial}{\partial t} P_{A}=P_{A}^{2}+P_{B} P_{A}+P_{A} P_{B}-P_{A} \\
& \frac{\partial}{\partial t} P_{A}=P_{B}^{2}+P_{C} P_{B}+P_{B} P_{C}-P_{B} \\
& \frac{\partial}{\partial t} P_{C}=P_{C}{ }^{2}+P_{A} P_{C}+P_{C} P_{A}-P_{C} .
\end{aligned}
$$

(4) can be derived more rigorously from the master equation on the analogy of the way in the book of M. Kac [1].

The collision rule can be represented by the following algebraic structure,

$$
\begin{array}{ll}
E_{A} \circ E_{A}=E_{A} & E_{A} \circ E_{B}=E_{B} \circ E_{A}=E_{A} \\
E_{B} \circ E_{B}=E_{B} & E_{B} \circ E_{C}=E_{C} \circ E_{B}=E_{B} \\
E_{C} \circ E_{C}=E_{C} & E_{C} \circ E_{A}=E_{A} \circ E_{C}=E_{C} .
\end{array}
$$

We can consider a linear space $P_{A} E_{A}+P_{B} E_{B}+P_{C} E_{C}$, which represents the state of the system.

Define

$$
\left(P_{A} E_{A}+P_{B} E_{B}+P_{C} E_{C}\right) \circ\left(P_{A}^{\prime} E_{A}+P_{B}^{\prime} E_{B}+P_{C}^{\prime} E_{C}\right)
$$

so as to satisfy the distribution law and (5), we find that the product means "quasi-convolution" on a algebraic structure (5). Since the operation is not associative, we are not able to say "convolution".

By (5) and (6), (4) takes the form

$$
\begin{aligned}
\frac{\partial}{\partial t}\left(P_{A} E_{A}+P_{B} E_{C}+P_{C} E_{C}\right) & \\
= & \left(P_{A} E_{A}+P_{B} E_{B}+P_{C} E_{C}\right) \circ\left(P_{A} E_{A}+P_{B} E_{B}+P_{C} E_{C}\right) \\
& -\left(P_{A} E_{A}+P_{B} E_{B}+P_{C} E_{C}\right) .
\end{aligned}
$$

Symbolically (7) is isomorphic to Boltzmann equation [3]

$$
\frac{\partial}{\partial t} u=u \circ u-u \text {. }
$$

$u \circ u$ may be also said "quasi-convolution". E. Wild's solution of Boltzmann's problem can be adapted to (7) as well as (8).

Now we consider the trajectory of (4). Since $P_{A}+P_{B}+P_{C}=1$

$$
\begin{aligned}
& \frac{\partial}{\partial t} P_{A}=P_{A}\left(P_{B}-P_{C}\right) \\
& \frac{\partial}{\partial t} P_{B}=P_{B}\left(P_{C}-P_{A}\right) \\
& \frac{\partial}{\partial t} P_{C}=P_{C}\left(P_{A}-P_{B}\right) .
\end{aligned}
$$

Accordingly

$$
\frac{1}{P_{A}} \frac{\partial}{\partial t} P_{A}+\frac{1}{P_{B}} \frac{\partial}{\partial t} P_{B}+\frac{1}{P_{C}} \frac{\partial}{\partial t} P_{C}=0 .
$$


So

$$
\frac{\partial}{\partial t} \log P_{A} P_{B} P_{C}=0 \text {. }
$$

The trajectory is on the solution of the following [2]

$$
\begin{aligned}
P_{A}+P_{B}+P_{C} & =1 \\
P_{A} \cdot P_{B} \cdot P_{C} & =k_{1} .
\end{aligned}
$$

\section{Model II.}

1), 2) are the same to Model I.

3) If an $A$ collides with a $B$, the $A$ varies to a $B$ with probability $1-P_{A B}$ and remains unchanged with probability $P_{A B}$, while the $B$ varies to an $A$ with probability $P_{A B}$ and remains unchanged with probability $1-P_{A B}$. In other words, $E_{A} \circ E_{B}=E_{B} \circ E_{A}=P_{A B} E_{A}+\left(1-P_{A B}\right) E_{B}$.

The collision between the same types makes no change. For another combinations, the system is ruled by similar laws.

If $n$ is sufficiently large, it can be formulated as a Boltzmann eqation (7) on a randomized algebraic structure.

$$
\begin{array}{ll}
E_{A} \circ E_{A}=E_{A} & E_{A} \circ E_{B}=E_{B} \circ E_{A}=P_{A B} E_{A}+\left(1-P_{A B}\right) E_{B} \\
E_{B} \circ E_{B}=E_{B} & E_{B} \circ E_{C}=E_{C} \circ E_{B}=P_{B C} E_{B}+\left(1-P_{B C}\right) E_{C} \\
E_{C} \circ E_{C}=E_{C} & E_{C} \circ E_{A}=E_{A} \circ E_{C}=P_{C A} E_{C}+\left(1-P_{C A}\right) E_{A}
\end{array}
$$

The difference between Model I and Model II is caused by the algebraic structure.

From (7) and (13), we can derive

$$
\begin{aligned}
& \frac{1}{P_{A}} \frac{\partial}{\partial t} P_{A}=P_{A}+2 P_{B} P_{A B}+2 P_{C}\left(1-P_{C A}\right)-1 \\
& \frac{1}{P_{B}} \frac{\partial}{\partial t} P_{B}=P_{B}+2 P_{C} P_{B C}+2 P_{A}\left(1-P_{A B}\right)-1 \\
& \frac{1}{P_{C}} \frac{\partial}{\partial t} P_{C}=P_{C}+2 P_{A} P_{C A}+2 P_{B}\left(1-P_{B C}\right)-1 .
\end{aligned}
$$

We can reform (14) to

$$
\begin{aligned}
& \frac{1}{P_{A}} \frac{\partial}{\partial t} P_{A}=\quad P_{B}\left(2 P_{A B}-1\right)+P_{C}\left(1-2 P_{C A}\right) \\
& \frac{1}{P_{B}} \frac{\partial}{\partial t} P_{B}=P_{A}\left(1-2 P_{A B}\right) \\
& \frac{1}{P_{C}} \frac{\partial}{\partial t} P_{C}=P_{A}\left(2 P_{C A}-1\right)+P_{B}\left(1-2_{B C}\right) .
\end{aligned}
$$

Consider

$$
\alpha \frac{1}{P_{A}} \frac{\partial}{\partial t} P_{A}+\beta \frac{1}{P_{B}} \frac{\partial}{\partial t} P+\gamma \frac{1}{P_{C}} \frac{\partial}{\partial t} P_{C} .
$$

For appropriate time independent constants $\alpha, \beta, \gamma$, we can make (16) equal zero.

Because (17) has non-trivial solution. 


$$
\begin{aligned}
\left(1-2 P_{A B}\right) \beta+\left(2 P_{C A}-1\right) \gamma & =0 \\
\left(2 P_{A B}-1\right) \alpha+\left(1-2 P_{B C}\right) \gamma & =0 \\
\left(1-2 P_{C A}\right) \alpha+\left(2 P_{B C}-1\right) \beta & =0 .
\end{aligned}
$$

For

$$
\left(\begin{array}{ccc}
0 & 1-2 P_{A B} & 2 P_{C A}-1 \\
2 P_{A B}-1 & 0 & 1-2 P_{B C} \\
1-2 P_{C A} & 2 P_{B C}-1 & 0
\end{array}\right)
$$

is a skew symmetric matrix, the determinant is zero. (The determinant of an odd dimensional skew symmetric matrix is zero.)

The trajectory is on the solution of

$$
\begin{aligned}
P_{A}^{\alpha} P_{B}^{\beta} P_{C}^{r} & =k_{2} \\
P_{A}+P_{B}+P_{C} & =1 .
\end{aligned}
$$

By $(\alpha, \beta, \gamma)$, many types of trajectory can be considered.

The trajectory of Model II is on a curve on $P_{A}+P_{B}+P_{C}=1\left(P_{A}\right.$, $\left.P_{B}, P_{C}>0\right)$.

The author made Model II from an actual problem of operations research which was suggested by Prof. K. Kunisawa.

This type of phenomena often occurs in economics, for example, the change of the market share.

In this case collision means comparison, and uniform distribution of comparing pairs is assumed.

We can extend the above discussion to the struggle for existence among $n=2 k+1$ species.

It will be formulated as a Boltzmann equation (20) on a randomized algebraic structure (21).

$$
\begin{aligned}
\frac{\partial}{\partial t}\left(\sum_{i=1}^{n} P_{i} E_{i}\right) & =\left(\sum_{i=1}^{n} P_{i} E_{i}\right) \circ\left(\sum_{i=1}^{n} P_{i} E_{\imath}\right)-\left(\sum_{i=1}^{n} P_{i} E_{i}\right) \\
E_{i} \cdot E_{j} & =P_{i j} E_{i}+\left(1-P_{i j}\right) E_{j} \\
P_{i j} & =P_{j i} \\
i, j & =1,2, \cdots, n, \quad n=2 k+1 .
\end{aligned}
$$

For appropriate time independent constants $\alpha_{1}, \alpha_{2}, \cdots, \alpha_{n}$, the trajectory satisfies

$$
\begin{aligned}
& \sum_{i=1}^{n} P_{i}=1 \\
& \sum_{i=1}^{n} P_{i}^{\alpha}=k_{3} \quad n=2 k+1 .
\end{aligned}
$$

Acknowledgement. I am much indebted to Prof. K. Kunisawa for his valuable suggestion and advice. Prof. H. Tanaka has kindly taught me his method about Boltzmann-type problem. Dr. Y. Horibe and Mr. T. Fujimagari have encouraged me throughout the preparation of this paper. 


\section{References}

[1] Kac, M.: Probability and Related Topics in Physical Sciences. New York (1959).

[2] Kimura, M.: Mathematical Theory of Genetics. Iwanami-Koza, GendaiOyo-Sugaku, Tokyo (1957) (in Japanese).

13 ] Mckean, H. P. Jr.: Speed of approach to equilibrium for Kac's caricature of Maxwellian gas. Arch. Rational Mech. Anal., 21, 343-367 (1966).

[4] Volterra, V.: Leçons sur la théorie mathématique de la lutte pour la vie. Cahiers scientifiques. VII. Paris, Gauthier-Villas (1931). 